\title{
A randomized trial of the effects of the no-carrageenan diet on ulcerative colitis disease activity
}

\author{
Sumit Bhattacharyya ${ }^{\mathrm{a}, \mathrm{b}}$, Theresa Shumard ${ }^{\mathrm{c}}$, Hui Xie ${ }^{\mathrm{d}}$, Amar Dodda ${ }^{\mathrm{a}, 1}$, Krista A. Varady ${ }^{\mathrm{e}}$, \\ Leo Feferman ${ }^{\mathrm{a}, \mathrm{b}}$, Allan G. Halline ${ }^{\mathrm{a}}$, Jay L. Goldstein ${ }^{\mathrm{a}, 2}$, Stephen B. Hanauer ${ }^{\mathrm{c}, 3}$ \\ and Joanne K. Tobacman ${ }^{\mathrm{a}, \mathrm{b}, *}$ \\ ${ }^{a}$ Department of Medicine, University of Illinois at Chicago, Chicago, IL, USA \\ ${ }^{\mathrm{b}}$ Jesse Brown VA Medical Center, Chicago, IL, USA \\ ${ }^{\mathrm{c}}$ Department of Medicine, University of Chicago, Chicago, IL, USA \\ ${ }^{\mathrm{d}}$ Division of Epidemiology and Biostatistics, University of Illinois at Chicago, Chicago, IL and Faculty of Health \\ Sciences, Simon Fraser University, Burnaby, BC, Canada \\ ${ }^{\mathrm{e}}$ Department of Kinesiology and Nutrition, University of Illinois at Chicago, Chicago, IL, USA
}

\begin{abstract}
. thousands of cell-based and animal experiments. remission. placebo-group. of dietary carrageenan may benefit patients with ulcerative colitis.

\footnotetext{
${ }^{1}$ Medical College of Wisconsin, Milwaukee, WI, USA.

${ }^{2}$ NorthShore University HealthSystem, Evanston, IL, USA.

${ }^{3}$ Northwestern University, Chicago, IL, USA.

${ }^{*}$ Corresponding author: Joanne K. Tobacman, M.D., University of Illinois at Chicago, 840S. Wood St., CSN 440, Chicago, IL 60612, USA. Tel.: +1 3125697826 ; Fax: +1 312413 8283; E-mail: jkt@uic.edu.
}

BACKGROUND: Carrageenan is a very common food additive in Western diets, but predictably causes inflammation in

OBJECTIVE: To assess the impact of carrageenan exposure on the interval to relapse in patients with ulcerative colitis in

METHODS: A randomized, double-blind, placebo-controlled, multicenter, clinical trial was conducted to assess if patients with ulcerative colitis in remission would have a longer interval to relapse if they followed a diet with no carrageenan. All participants were instructed in the no-carrageenan diet and were randomized to either placebo capsules or carrageenancontaining capsules. The carrageenan in the capsules was less than the average daily carrageenan intake from the diet. Relapse was defined as an increase of two or more points on the Simple Clinical Colitis Activity Index (SCCAI) and intensification of treatment for ulcerative colitis. Participants were followed by telephone calls every two weeks until relapse or one year of participation. The occurrence of relapse and inflammatory biomarkers were compared between the two groups.

RESULTS: Twelve patients completed study questionnaires. Three patients who received carrageenan-containing capsules relapsed, and none of the patients who received placebo-containing capsules relapsed ( $p=0.046$, log-rank test). Laboratory tests showed increases in Interleukin-6 ( $p=0.02$, paired $t$-test, two-tailed) and fecal calprotectin $(p=0.06$; paired $t$-test, two-tailed) between the beginning and the end of study participation in the carrageenan-exposed group, but not in the

CONCLUSION: Carrageenan intake contributed to earlier relapse in patients with ulcerative colitis in remission. Restriction

Keywords: Colitis, carrageenan, inflammation, food additive, Interleukin-6 


\section{List of abbreviations}

\begin{tabular}{|c|c|}
\hline $\begin{array}{l}\text { BCL } \\
\text { CGN }\end{array}$ & $\begin{array}{l}\text { B-cell leukemia/lymphoma } \\
\text { carrageenan }\end{array}$ \\
\hline CONSORT & $\begin{array}{l}\text { Consolidated Standards Of Reporting } \\
\text { Trials }\end{array}$ \\
\hline IBD & inflammatory bowel disease \\
\hline IL & interleukin \\
\hline $\mathrm{MCP}$ & monocyte chemoattractant protein \\
\hline NF-кB & nuclear factor kappa B \\
\hline N.D. & no difference \\
\hline S.D. & standard deviation \\
\hline SCCAI & Simple Clinical Colitis Activity Index \\
\hline SIBDQ & $\begin{array}{l}\text { Short Inflammatory Bowel Disease } \\
\text { Questionnaire }\end{array}$ \\
\hline TNF & tumor necrosis factor \\
\hline $\mathrm{UC}$ & ulcerative colitis \\
\hline
\end{tabular}

\section{Introduction}

The common food additive carrageenan has been used in thousands of experiments in animal models and in cultured human cells to cause inflammation [1-8]. Carrageenan-induced inflammatory effects in the intestine in animal models resemble the histopathology of ulcerative colitis [6-8]. This predictable response to carrageenan has been used to identify chemical and cellular mediators of inflammation and to assess the effectiveness of antiinflammatory treatments [9-16]. The inflammatory cascades activated by carrageenan exposure are mediated by reactive oxygen species and by activation of an innate immune pathway involving the toll-like receptor (TLR)-4 and B-cell leukemia/lymphoma (BCL) 10 [17-20].

Carrageenan is a highly sulfated polysaccharide, composed of galactose residues which are linked in alternating beta-1,4 and alpha-1,3 bonds. Carrageenan resembles the naturally occurring galactose-containing sulfated glycosaminoglycans keratan sulfate and chondroitin sulfate, but differs by the presence of the unusual $\alpha-\operatorname{Gal}(1,3) \mathrm{Gal}$ bonds which are not innate to humans and are immunogenic [21-25]. Carrageenan occurs in three major varieties, kappa (к) lambda $(\lambda)$, and iota (८), which are derived from several species of red algae. Most of the food-grade carrageenan is comprised of $\kappa$ - and $\lambda$-carrageenans of molecular weight (MW) 200,000800,000 [3]. Both low and high molecular weight carrageenan has been shown to cause intestinal inflammation [2].
Carrageenan is routinely consumed in the typical Western diet, with average intake estimated to be about $250 \mathrm{mg} /$ day [26, 27]. Intake by some individuals may be as much as $2-4$ grams/day [28], and average daily intake of $1.08-7.2 \mathrm{~g} /$ day in a $60 \mathrm{~kg}$ person $(18-40 \mathrm{mg} / \mathrm{kg}$ day) was reported recently in an industry-sponsored study [29]. Carrageenan is incorporated into a wide variety of processed foods, including ice cream, sandwich meats, soymilk, yogurt, infant formula, dietetic beverages, and canned whipped cream, in order to improve the texture of the food product by increasing the solubility of ingredients and thickening the mixture.

Since carrageenan is consumed in sufficient quantity in the Western diet to induce inflammation, this investigation was designed to test how carrageenan intake might influence the clinical course in patients with ulcerative colitis. We hypothesized that withdrawal of carrageenan from the diet might have a role in delaying the occurrence of relapses in patients with ulcerative colitis in remission. To test whether consumption of carrageenan might contribute to ulcerative colitis relapse and whether a no-carrageenan diet study was feasible, we initiated a randomized, double-blind, multicenter, placebocontrolled trial in patients with ulcerative colitis in remission with a high risk of relapse.

\section{Materials and methods}

\subsection{Study design}

The investigation was designed as a doubleblind, randomized, multi-institutional clinical trial to compare the effect of the common food additive carrageenan on the interval to relapse in adult patients with biopsy-proven ulcerative colitis (UC) in clinical remission for at least one month prior to study entry. The study protocol was approved by the Institutional Review Boards of the University of Illinois at Chicago, the Jesse Brown VA Medical Center, and the University of Chicago. The study was supported by the Broad Medical Research Program. The study was registered on the ClinicalTrials.gov website ("Effects of Carrageenan-Elimination Diet on Ulcerative Colitis Disease Activity" NCT01065571). All participants provided written informed consent, but were not asked to give consent for publication of individual data. Data supporting study conclusions are included within this report. The investigators had no conflict of interest with regard to the study. 


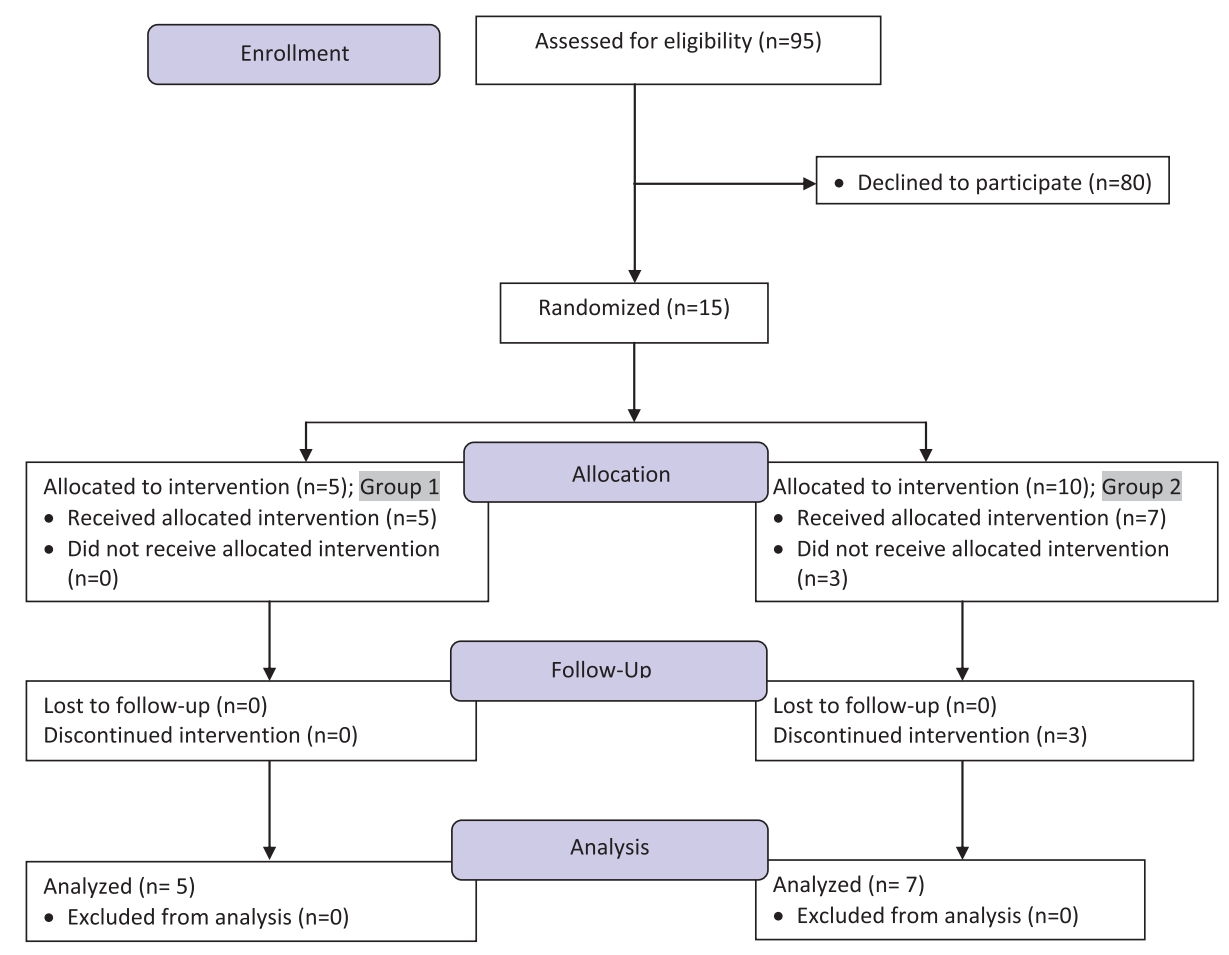

Fig. 1. Study flow diagram. This flow chart presents the number of subjects approached in enrollment, the number of subjects allocated to carrageenan or placebo capsules, and the number of participants in follow-up and analysis. Group 1 received the carrageenan-containing capsules, and Group 2 received similar-appearing placebo capsules.

Participants were recruited from December 2009 to December 2012, and study procedures ended in December, 2013. Entry criteria included: over 18 years of age; a biopsy-confirmed diagnosis of ulcerative colitis; previous need for corticosteroids to obtain remission; in clinical remission off corticosteroids for at least one month; SCCAI score of 2 or less; on no medications or stable dose of maintenance medication; and willingness to follow a carrageenan-free diet for 12 months. Exclusion criteria included: active UC on corticosteroids; unable to read labels on food products; inability to make choices about diet. CONSORT recommendations for transparent reporting of clinical trials were observed. The study flow diagram is presented in Fig. 1 [30], and the CONSORT checklist is included as Supplementary Table 1.

All participants were instructed by study investigators and/or participating dieticians in the carrageenan-free diet (Table 1). They agreed to follow the carrageenan-free diet for the duration of their participation in the study. Participants were required to be able to make their own food choices and to be able to read labels about ingredients in food products. Participants were randomized to receive either capsules containing $100 \mathrm{mg}$ of food-grade carrageenan or sim- ilar appearing dextrose-containing capsules. Subjects initially took one capsule daily, and increased intake to two capsules daily after finding that one capsule daily was well-tolerated. The amount of carrageenan in two capsules $(200 \mathrm{mg}$ ) was less than the anticipated daily consumption of carrageenan in the average adult diet in the United States $(250 \mathrm{mg})$ [26, 27]. Since no precise measurements of carrageenan in food products were available, the amount of carrageenan consumed when there was non-compliance with the no-carrageenan diet could not be calculated.

Throughout the study, participants received their medical care from their personal physicians, who were blinded to the treatment assignment. Patients continued on their maintenance medications and were not allowed to increase doses or add new therapies. Data in all study records were anonymized, and records were compiled with a code for identification.

The primary outcome measure was occurrence of relapse, which was defined as an increase of two (or more) points on the SCCAI [31] in association with an increase in treatment (either an increase in maintenance medication dose or addition of new therapies for flare) by the participant's personal physician. Relapse terminated study participation. Occurrences 
Table 1

List of foods to avoid in the no-carrageenan diet

Almond milk
Bakery products with glazes, frostings
Beer
Candy; chocolate candies
Canned fish, meats
Chocolate milk mixes/powders, condensed milk,
$\quad$ evaporated milk powders
Cottage cheese
Dietetic beverages
Deli meat
Evaporated milk, condensed milk, powdered milk,
flavored milk, flax milk
Frosting base mix, canned frostings
Gelled fruit snacks
Ice cream, frozen custard, frozen yogurt, sherbets, etc.
Infant formulas
Liquid coffee whitener
Maple Syrups
Meal Replacements
Nutritional drinks
Pie filling
Processed meats, fish or cheese
Pudding
Ricotta cheese
Soy Milks
Whipped cream (canned)
Yogurt

of relapse were compared between the two groups of patients at 3, 6, 9, and 12 months.

\subsection{Study procedures}

Adult patients with ulcerative colitis in clinical remission for at least one month and who required corticosteroids to go into remission previously were recruited from the Digestive Disease Center of the University of Illinois at Chicago (UIC) and in the Gastroenterology Clinic of the University of Chicago ( $\mathrm{U}$ of C). Other potential participants contacted study investigators, after seeing the notice about the trial on the ClinicalTrials.gov website [32]. Medical records were reviewed to document the biopsy-proven diagnosis of ulcerative colitis and eligibility for study participation. Subsequently, participants completed informed consent, HIPAA documentation, instruction in the no-carrageenan diet, blood and stool sample collection, completion of study questionnaires, and randomization to either carrageenan-containing capsules or placebo capsules. Two randomization schemes were used due to convenience. Randomization at UIC was performed by a UIC investigational pharmacist who had no contact with the study participants. Allocation to study tablets \#1 or \#2 was performed by using a randomization table (www.randomization.com) in which the next available treatment was assigned to the next study enrollee. Within a block of ten subjects, an equal number of assignments to \#1 or \#2 was allocated. Randomization at $\mathrm{U}$ of $\mathrm{C}$ was performed by a blinded investigator, who allocated assignment based on the participant's year of birth [odd or even; \#1 or \#2].

Subjects were given written and verbal instruction about the carrageenan-free diet (Table 1) by study investigators (TS or JKT) or by participating dieticians associated with the Clinical Research Center at UIC or the Gastroenterology Clinic at the $\mathrm{U}$ of $\mathrm{C}$. Recommendations included specific brands, when such information was available. The study follow-up questionnaires included detailed information about adherence to the carrageenan-free diet and compliance with taking study capsules. Two standardized questionnaires, the Short Inflammatory Bowel Disease Questionnaire (SIBDQ) [33] and the Simple Clinical Colitis Activity Index (SCCAI) [31], were used to assess disease status and quality of life.

Study participants completed questionnaires by telephone contact with study personnel every two weeks. Subjects indicated their dietary lapses at the time of the telephone contacts, and completed $24 \mathrm{~h}$ dietary recalls. Consumption of two or more carrageenan-containing foods in consecutive twoweek intervals was a criterion for removal from study participation. Subjects participated in the study for either one year, or until they withdrew or relapsed. Blood and stool samples were collected every three months, at which time unused capsules were returned and new bottles of capsules were issued. During the study, the protocol was amended to enable participants to submit stool samples by mail every three months, to forego the every three month blood draw, to return unused capsules by mail, and to receive a new supply of capsules by mail, rather than in person.

\subsection{Study capsules}

Carrageenan-containing capsules were prepared by the Chau Center for Industrial Pharmacy and Contract Manufacturing (West Lafayette, Indiana). Carrageenan capsules contained $100 \mathrm{mg}$ of foodgrade carrageenan obtained from FMC Biopolymer (Philadelphia, PA). Equal parts of kappa (Gelcarin; GP-812NF), lambda (Viscarin; GP-209NF), and iota (Gelcarin; GP-379NF) carrageenan were incorporated in a gelatin capsule. Placebo capsules 
containing dextrose (Sigma Chemical Co., St. Louis, $\mathrm{MO}$ ) were prepared under similar, rigorous qualitycontrolled conditions. Products passed microbial surveillance performed by Lancaster Laboratories (Lancaster, PA). Capsules were distributed to study participants in screw-top bottles of 100 capsules. Participants were advised to take the capsules with a meal to simulate consumption of carrageenan in processed foods.

\subsection{Study instruments: Simple Clinical Colitis Activity Index (SCCAI) and Short Inflammatory Bowel Disease Questionnaire (SIBDQ)}

The SCCAI was designed for use in the initial evaluation of exacerbations of ulcerative colitis [31]. Items on the SCCAI are scored from $0-4$, with a maximum score of 19. Items include: bowel frequency (day), bowel frequency (night), urgency of defecation, hematochezia, general well-being, and extracolonic disease manifestations, including arthritis, pyoderma gangrenosum, uveitis, and erythema nodosum. Scores were calculated based on biweekly responses to the questionnaires and compared to previous results to detect an increase of 2 or more points. The SCCAI correlates well with other IBD indices, including those of Seo et al [34] and Truelove [35].

The Short Inflammatory Bowel Disease Questionnaire [33] is a subjective measure of quality of life and of the influence of disease activity on daily life. It contains ten questions answered on a scale from 1-7, with 7 indicating all of the time and 1 indicating never. The questions address health status over the previous two weeks and include: feeling of fatigue; effect of IBD on social and sexual activity; effect of IBD on leisure or sports activity; abdominal pain, depression/discouragement; passing large amount of gas; maintaining weight; relaxed/free of tension; feeling of tenesmus; and feeling of anger.

\subsection{Laboratory tests}

Blood samples for inflammatory parameters, including serum Interleukin(IL)-8, Interleukin (IL)-6, Tumor Necrosis Factor (TNF)- $\alpha$, monocyte chemoattractant protein (MCP) -1 , leukocyte Nuclear Factor(NF)-kappaB (p65; NF- $\kappa \mathrm{B})$ and B-cell leukemia/lymphoma (BCL)10, and stool sample for calprotectin ( $\& \& D$, Minneapolis, $M N$ ) were obtained at entry into the study, at three-month intervals (unless exempted as above), and/or at termination of study participation. Serum was separated and frozen for subsequent ELISA assays for IL-6 (R\&D), IL-8 (R\&D), MCP-1 (R\&D) and TNF- $\alpha$ (R\&D). Peripheral leukocytes were isolated from whole blood (Polymorphprep ${ }^{\mathrm{TM}}$, Axis-Shield, Inc., Norton, MA) at the time of blood sample collection and frozen for subsequent ELISA assays for $\mathrm{NF}-\kappa \mathrm{B}(\mathrm{R} \& \mathrm{D})$ and BCL10 [36]. Samples were frozen and batched, and assays were performed at regular intervals throughout the study, without knowledge of participant assignment to Group 1 or Group 2. Assays followed the manufacturer's recommended procedures, with technical replicates and known standards.

\subsection{Statistical analysis}

Laboratory tests were performed with technical replicates and mean values were calculated for each sample; then, mean values with standard deviation were calculated for each group. Significance of differences between groups was calculated by unpaired $t$-tests, two-tailed, with $p<0.05$ designated as significant. Differences between individual results at different time points were determined by paired $t$-tests, two-tailed, with $p<0.05$ as significant. Values were calculated for data at entry, at interim intervals, and at conclusion of participation. SCCAI and SIBDQ scores were calculated for each participant at each time point (q2wk). Results were compared for each participant with their prior scores and between groups.

Original sample size calculations estimated that a total of 36 subjects (18/group) would be necessary to achieve $80 \%$ power to detect a SCCAI score difference of 1.0 between the groups. This was based on the assumption that the mean SCCAI score in the carrageenan-consuming group would be at least 2 and the mean score in the carrageenan-free group would be 1, with standard deviations of 1.0. Study biostatistician (HX) was unblinded as to participant assignment in order to perform the data analysis, and did not communicate with investigators who had patient contact. The log-rank test was performed at six-month intervals to detect if there were significant differences between the two study arms in the frequency of relapse (PROC LIFETEST, SAS 9.3, Cary, NC). These differences are reported in the KaplanMeier life table analysis. The study was terminated when a statistically significant difference between the two study groups was detected by the log-rank test. 
Table 2

Characteristics of study groups

\begin{tabular}{lll}
\hline Characteristic & $\begin{array}{l}\text { Carrageenan- containing } \\
\text { Capsules (Group 1) }\end{array}$ & Placebo Capsules (Group 2) \\
\hline Female & 2 & 2 \\
Male & 3 & 5 \\
Age Range; & $43-65$ & $34-64$ \\
Mean Age & 54 & 50 \\
Relapses & 3 & 0 \\
Dropouts & 0 & 3 \\
Race & Caucasian (4) & Caucasian (4) \\
& African-American (1) & African-American (2) \\
Medications at the time of & & Indian (1) \\
entry into the study & None & Asacol, Azathioprine, Humira \\
& None & Asacol \\
& Imuran & Sulfsalazine Sulfsalazine \\
& Asacol, Humira & Sulfsalazine, Purinethol \\
& Asacol, Humira & Imuran \\
& & Asacol \\
\hline
\end{tabular}

\section{Results}

\subsection{Study participants}

Fifteen patients enrolled in the study, but three declined further participation following randomization, citing reluctance to comply with study procedures. Twelve participants followed the nocarrageenan diet, and five patients were randomized to carrageenan-containing capsules (Group 1) and seven patients were randomized to placebo (Group 2). Characteristics of the participants are presented in Table 2. Participants were ethnically diverse, with similar age and gender distribution

All participants reported occasional intake of carrageenan-containing foods and occasional lapses in taking capsules. No subjects met the criterion for removal from the study due to non-adherence to the no-carrageenan diet. The 24-hour dietary recalls demonstrated an overall high level of adherence to the no-carrageenan diet. Non-compliance with the study protocol, including ingestion of carrageenancontaining foods and lapses in taking the study capsules, occurred, but the frequency of these events was less than $10 \%$ of total study days for all participants.

\subsection{SCCAI and SIBDQ scores}

Mean SCCAI (Fig. 2A) and SIBDQ (Fig. 2B) scores were similar at onset between the two groups, indicating the comparability of the two groups at baseline. Mean SCCAI score at onset was $1.29 \pm 1$ in the placebo control group and was $1.20 \pm 0.84$ in the
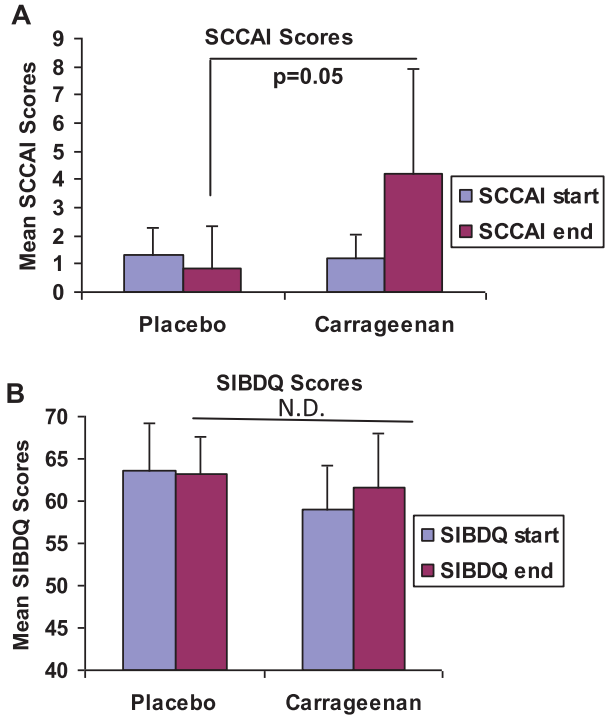

Fig. 2. Average SCCAI and SIBDQ scores for placebo and carrageenan-supplemented groups. (A) Mean SCCAI scores were similar for both groups at the onset of study participation. At the conclusion of participation, SCCAI scores were higher in the carrageenan-supplemented group, consistent with the occurrence of relapses in this group. Mean SCCAI scores at the endpoints were $0.86 \pm 1.46(n=7)$, compared to $4.20 \pm 3.70$ in the group who received carrageenan supplements ( $p=0.05$, unpaired $t$-test, two-tailed, $n=5$ ). (B) Initial and final scores on the SIBDQ were similar in both groups, with mean values of $63.6 \pm 5.6$ at onset and $63.3 \pm 4.3$ at conclusion in the placebo group $(n=7)$. In the carrageenan- supplemented group $(n=5)$, initial mean score was $59.0 \pm 5.2$, and final average score was $61.6 \pm 6.5$.

carrageenan-exposed group. At the conclusion, the scores were $4.20 \pm 3.70$ in the carrageenan group and $0.86 \pm 1.46$ in the placebo group $(p=0.05$, unpaired 


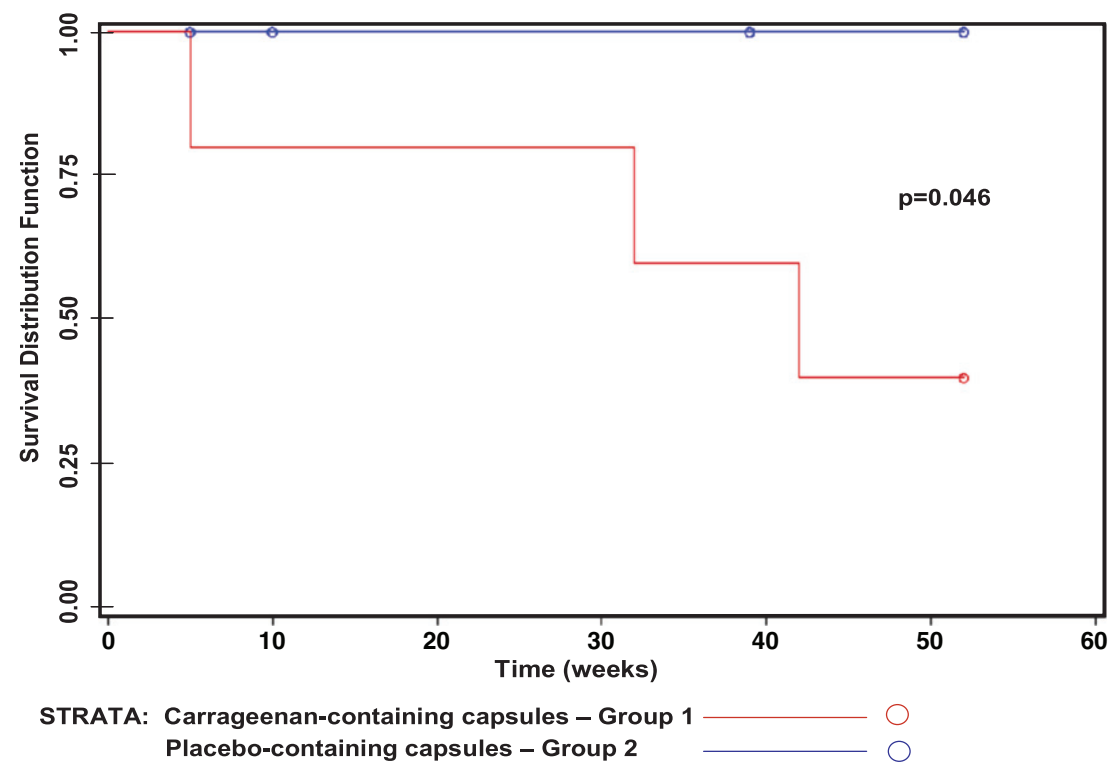

Fig. 3. Kaplan-Meier curves comparing the carrageenan-supplemented group (Group 1) and the placebo group (Group 2). Kaplan-Meier curves indicate significant differences between the two study groups. Three dropouts in the control group occurred, at 5, 10, and 39 weeks. Relapses in the carrageenan supplement group occurred at 5, 32, and 42 weeks.

$t$-test, two-tailed). Scores increased by two or more points in the three patients in the carrageenan group who relapsed (to 5, 6, and 9), and in one patient in the placebo group. The patient in the placebo group did not have an increase in his colitis regimen and subsequent sigmoidoscopy did not show an increase in his colitis. The difference in the mean SCCAI scores between the two groups was substantially higher than the estimate of a difference of 2 between the two groups which was used in the sample size calculation.

Scores on the SIBDQ (Fig. 2B) were similar in both groups, and ranged from 52 to 68 at the start, and from 51 to 70 at the end of participation.

\subsection{Relapses in patients on carrageenan supplements}

The primary outcome measure was occurrence of relapse, which was defined as an increase of two or more points on the SCCAI [31], in association with an increase in treatment (either an increase in maintenance medication dose or addition of new therapies for flare) by the participant's personal physician for manifestations of ulcerative colitis. Kaplan-Meier Life-Table curves indicated that the carrageenansupplemented treatment group was more likely to have relapses than the placebo group $(p=0.046$, log-rank test) (Fig. 3). Twelve subjects had either completed one year of study procedures, relapsed, or withdrawn at the time of this analysis, and no additional patients were recruited following this determination of significant difference between the two groups. By unpaired $t$-test, two-tailed, study results were not yet statistically significant.

Relapses, defined as an increase in two or more points on the SCCAI in association with increase in treatment for manifestations of UC by the participant's physician, occurred at 5 weeks, 32 weeks, and 42 weeks in three subjects who were receiving carrageenan supplements. Relapses were manifested by hematochezia in two subjects and by joint inflammation in one subject in whom joint inflammation had previously been considered as a manifestation of UC. Two patients who relapsed were on two medications, and one patient was on no treatment for UC at the time of relapse. Two participants in the carrageenan arm completed 52 weeks of the clinical trial without relapse. No relapses occurred in the participants who received placebo capsules, four of whom completed 52 weeks of participation. One patient in the placebo arm had an increase in SCCAI of greater than two points, but did not have any increase in treatment by his physician, so did not meet criteria for relapse. Four patients on the no-carrageenan diet and taking placebo capsules had decline in their SCCAI scores. 
Table 3

Measurements of inflammatory parameters at onset and conclusion of participation in placebo and carrageenan study groups

\begin{tabular}{|c|c|c|c|c|}
\hline $\begin{array}{l}\text { Inflammatory } \\
\text { Parameter } \\
\text { Value } \pm \text { S.D. }\end{array}$ & $\begin{array}{c}\text { Group } 1 . \\
\text { Carrageenan-containing } \\
\text { capsules - onset }\end{array}$ & $\begin{array}{c}\text { Group } 1 . \\
\text { Carrageenan-containing } \\
\text { capsules - conclusion }\end{array}$ & $\begin{array}{c}\text { Group } 2 . \\
\text { Placebo capsules } \\
\text { - onset }\end{array}$ & $\begin{array}{c}\text { Group } 2 . \\
\text { Placebo capsules } \\
\text { - conclusion }\end{array}$ \\
\hline IL-6 (pg/ml) & $2.57(0.97)$ & $5.00(2.31)^{\mathrm{a}}$ & $3.69(1.68)$ & $3.06(1.44)$ \\
\hline Calprotectin $(\mu \mathrm{g} / \mathrm{gm})$ & $133(125)$ & $171(143)^{b}$ & 149 (112) & $111(91)$ \\
\hline IL-8 (pg/ml) & $266(307)$ & $207(180)$ & $151(65)$ & 169 (109) \\
\hline $\mathrm{TNF}-\alpha(\mathrm{pg} / \mathrm{ml})$ & $3.8(0.9)$ & $6.3(4.9)$ & $21.0(25.2)$ & $10.8(10.4)$ \\
\hline MCP-1 (pg/ml) & $161(84)$ & $188(157)$ & $55(26)$ & $107(46)$ \\
\hline $\begin{array}{l}\text { NF- } \kappa \mathrm{B}(\% \text { normal } \\
\text { control WBC) }\end{array}$ & $214(159)$ & $192(97)$ & $165(45)$ & $171(66)$ \\
\hline BCL10 (ng/ml) & $2.02(0.73)$ & $1.85(0.36)$ & $1.83(0.64)$ & $1.98(0.61)$ \\
\hline
\end{tabular}

a) for $p=0.02$, paired $t$-test, two-tailed; $n=5$, b) for $p=0.06$, paired $t$-test, two-tailed; $n=5, \mathrm{BCL}=\mathrm{B}$-cell leukemia/lymphoma; $\mathrm{IL}=$ Interleukin $; \mathrm{MCP}=$ monocyte chemoattractant protein; $\mathrm{NF}-\kappa \mathrm{B}=$ nuclear factor kappaB; $\mathrm{S} . \mathrm{D} .=$ standard deviation; $\mathrm{TNF}=$ tumor necrosis factor.
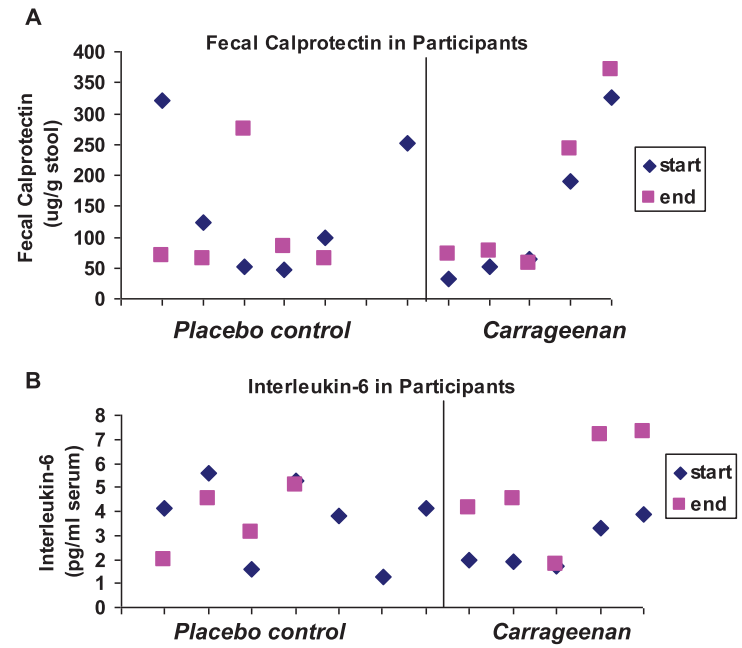

Fig. 4. Scatterplot of fecal calprotectin and Interleukin-6. (A) The initial and final values for fecal calprotectin are shown in the scatterplot by placebo controls and carrageenan-exposed participants. Two of the controls and four of the carrageenan-exposed subjects had increases $(p=0.06$ for carrageenan-exposed group, paired $t$-test, two-tailed; $n=5$ ). (B) Interleukin-6 values for the study subjects increased in all of the carrageenan-exposed participants and in one of the control subjects $(p=0.02$ for carrageenan-exposed group, paired $t$-test, two-tailed; $n=5$ ).

\subsection{Laboratory data}

Baseline mean laboratory values, including fecal calprotectin, serum Interleukin IL)-8, Interleukin (IL)-6, monocyte chemoattractant protein (MCP)-1, and tumor necrosis factor (TNF)- $\alpha$, and leukocyte NF-кB and BCL10 were not statistically different between the two groups and the standard deviations were large (Table 3 ). The similar values for these inflammatory parameters reflected the comparability of the two groups. In the carrageenan-exposed group, fecal calprotectin (Fig. 4A) and IL-6 (Fig. 4B) increased from study onset to conclusion of participation ( $p=0.06, p=0.02$, respectively; paired $t$-test, two-tailed). One of the patients in the carrageenanexposed group who had increases in fecal calprotectin and IL-6 was on no anti-inflammatory treatment. TNF- $\alpha$ levels declined in two of the control and in one of the carrageenan-exposed patients during the course of the study. There were no significant changes in the inflammatory parameters in the placebo group by paired or unpaired $t$-tests.

\section{Discussion}

The study findings indicate that carrageenan consumption may aggravate ulcerative colitis (UC) disease activity and reduce the interval to relapse in patients who are in clinical remission. Due to the small sample size, the study findings may be attributable to a type I error. However, in view of the larger than anticipated difference in the mean SCCAI scores between the groups at conclusion of study participation $(0.86 \pm 1.46$ for placebo vs. $4.20 \pm 3.70$ for carrageenan), a smaller sample size than was originally proposed $(n=36)$ could have been targeted. The study was curtailed when statistical significance in relapses between the two groups was demonstrated by the log-rank test, a better measure of the overall difference in relapses between the groups than the unpaired $t$-test. Since the daily consumption of carrageenan in the typical diet is higher than the carrageenan intake in this trial, the study findings are relevant to the clinical management of UC and to further clinical investigation of the impact of carrageenan consumption on the manifestations of UC. 
The signs/symptoms in UC patients may be attributable to some extent to ongoing exposure to carrageenan, as well as to other pro-inflammatory exposures. Although genetic factors and immune responses to bacterial products have been emphasized with regard to the pathogenesis of IBD, these study data suggest that carrageenan exposure may have a role in exacerbations of UC. The impact of carrageenan may be due to its ability to activate the pathway of innate immunity in the intestinal epithelial cells and to interact with the intestinal microbiota and to stimulate immune responses.

The increases in the inflammatory parameters (IL-6 and fecal calprotectin) in the carrageenanexposed participants suggest that a genuine difference in disease status occurred between the two study groups. The decline in TNF- $\alpha$ values during the course of the study in two of the control patients suggests that the no-carrageenan diet may have been beneficial for these participants. The overall interpretation of the laboratory data may be confounded by intercurrent infections or other inflammatory conditions, such as tendinitis in one control subject who resumed jogging during the study.

The etiology of UC is attributed to an exaggerated, genetically-determined, dysregulated immune response to endogenous microbiota or bacterial products in the intestinal lumen [37, 38]. The effects of carrageenan ingestion on the microbiota or on immunity were not addressed in this study, and this trial did not consider whether or not ingestion of carrageenan can contribute to the initial development of ulcerative colitis or to specific disease manifestations. The deleterious effects of carrageenan on relapse may be due to interaction of carrageenan with the microbiome and specific immune responses, as well as to the direct activation by carrageenan of inflammatory pathways in the colonic epithelial cells.

The administration of carrageenan in gelatin capsules with meals differs from ingestion of carrageenan in combination with protein, carbohydrate, fat, and other components in a processed food as part of the diet. However, the mechanisms of digestion are expected to hydrolyze chemical bonds and to metabolize the protein and fat components of food products, and to thereby separate carrageenan from the nutritive food substances, thus minimizing the impact of carrageenan encapsulation, as used in our study. Since a specific blood or urine test to detect the presence of carrageenan is not available at this time, precise measurements of compliance with the no-carrageenan diet could not be performed.
Recruitment of study participants was more difficult than anticipated due to several factors. The major factor was the reluctance of potential enrollees to be randomized to an intervention that might hasten relapse of their ulcerative colitis. The duration of the study (up to one year) was also an obstacle to recruitment of more participants. The requirement for adherence to a restricted diet for a prolonged period of time was a major impediment to enrollment for some potential study subjects, who wanted to continue to eat foods that they habitually ate. Also, the intensity and frequency of study procedures and followup were factors that hindered recruitment. The study required frequent interactions with study personnel (q2 wk telephone calls and q3 mo sample collections), which some potential recruits found burdensome.

Although dietary factors have been examined for their possible role in IBD [39-47], this is the first report to show potential deleterious effects on UC relapse by a specific food additive. The association of IBD with the Western diet and the increased prevalence of IBD in recent decades [48-51] correlate with increased carrageenan consumption [52, 53] and suggest that restriction of dietary carrageenan intake might help to reduce the incidence of IBD, as well as the frequency of UC relapses. Self-reports of improvement in gastrointestinal symptoms following reduction of carrageenan intake were recorded in an online questionnaire by the Cornucopia Institute, a public interest group concerned with diet. In response to their advocacy, over 47,000 individuals signed a petition to the National Organic Standards Board to remove carrageenan from the list of additives approved for inclusion in organic foods in the United States [54]. Reports as these suggest that adverse effects of carrageenan consumption may be common in the general population.

The Specific Carbohydrate Diet has also been used in the management of IBD $[55,56]$. The Specific Carbohydrate Diet does not allow for the consumption of grains, simple sugars (except for honey), dairy products or processed foods. As such, patients consume primarily meats, fish, nuts, eggs, vegetables and lowsugar fruits. This regimen has been shown to decrease intestinal inflammation by restoring the balance of bacteria within the gut and potentially resolving dysbiosis [57]. The carrageenan-free diet is similar to the Specific Carbohydrate Diet in that both diets exclude most dairy products and processed foods. These diets differ, however, in that the carrageenanfree diet does permit the consumption of grains and simple sugars. Whether the carrageenan-free diet 
also has a beneficial impact on the gut microbiome, despite the inclusion of grains, warrants further investigation.

The Mediterranean diet may also be effective in the management of IBD [58]. This diet emphasizes the consumption of fish, fruits, vegetables, whole grains, legumes, olive oil and nuts, while de-emphasizing the consumption of dairy products (particularly butter), and processed foods. This diet pattern has been shown to lower intake of n-6 fatty acids by $\sim 30 \%$, which alters the n-3 to n- 6 fatty acid ratio in a way that decreases the formation of inflammatory mediators implicated in IBD progression [59, 60]. The carrageenan-free diet is similar to the Mediterranean diet in that it limits the consumption of processed foods and dairy products. It can be speculated that the carrageenan-free diet may also decrease $n-6$ fatty acid intake by limiting the consumption of processed foods, which are high in soybean oil. The impact of the carrageenan-free diet on ratio of n-3 to $n-6$ fatty acids should be examined in future trials to see if some of its anti-inflammatory effects are also mediated via this mechanism.

\section{Author contributions}

Drs. Bhattacharyya and Feferman performed laboratory tests. Drs. Halline, Goldstein, Dodda, and Hanauer identified participants. Dr. Xie performed statistical analysis. Ms. Shumard enrolled patients and followed them throughout the study procedures. Dr. Varady evaluated the no-carrageenan diet and compared it with other diets. Dr. Tobacman supervised the recruitment, laboratory procedures, data accrual, and study personnel at UIC. Dr. Hanauer supervised Ms. Shumard at the University of Chicago. Drs. Tobacman and Hanauer wrote the manuscript.

\section{Acknowledgments}

The authors acknowledge the contributions of the University of Illinois at Chicago Clinical Research Center staff for data and specimen collection. Lori Welstead, MS, RD, LDN, at the University of Chicago and Amanda Guide, RD, at the University of Illinois at Chicago, consulted about the study diet and about specific food choices. Michael J. Pacini, Pharm.D., Investigational Pharmacist at the University of Illinois at Chicago, assisted with dispensing study capsules and with randomization of study participants. The Cornucopia Institute provided access to the questionnaire data that was collected on their website. The content is solely the responsibility of the authors and does not necessarily represent the official views of the sponsors. The full protocol can be accessed by communication with study investigators.

\section{Conflict of interest}

The authors have no conflicts of interest with regard to the content of this report.

\section{Supplementary material}

Supplementary material can be found here: http:// dx.doi.org/10.3233/NHA-170023.

\section{References}

[1] IARC Working Group on the Evaluation of the Carcinogenic Risk of Chemicals to Humans, Carrageenan. IARC monogr. Eval Carcino Risk Hum Lyon. 1983;31: 79-94.

[2] Tobacman JK. Review of harmful gastrointestinal effects of carrageenan in animal experiments. Environ Health Perspect. 2001;109:983-94.

[3] Blakemore WR, Harrell AR. Carrageenan. In: Meson A, Ed. Food Stabilizers, Thickeners, and Gelling Agents, Chapter 5. Hoboken: Wiley-Blackwell, Blackwell Publishing Ltd., 2011, pp. 73-94.

[4] Campo VL, Kawano DF, da Silva DB Jr, Carvalho I. Carrageenans: Biological properties, chemical modifications and structural analysis - A review. Carbohydr Polym. 2009;77(2):167-80.

[5] Liu J, Zhan X, Wan J, Wang Y, Wang C. Review for carrageenan-based pharmaceutical biomaterials. Favourable physical features versus adverse biological effects. Carbohydr Polym. 2015;121:27-36.

[6] Sharratt M, Grasso P, Carpanini F, Gangolli SD. Carrageenan ulceration as a model for human ulcerative colitis. Lancet. 1971;1(7691):192-3.

[7] Kim H-S, Berstad A. Experimental colitis in animal models. Scand J Gastroenterol. 1992;27:529-37.

[8] Onderdonk AB. The carrageenan model for experimental ulcerative colitis. Prog Clin Biol Res. 1985;186:237-45.

[9] Jaysekhar P, Rao SB, Santhakumari G. Effect of 5substituted benzylideneaminosalicylic acid on carrageenaninduced ulcerative colitis. Boll Chim Farm. 2004;143(8): 309-13.

[10] Goto M, Okamoto Y, Yamamoto M, Aki H. Antiinflammatory effects of 5-aminosalicylic acid conjugates with chenodeoxycholic acid and ursodeoxycholic acid on carrageenan-induced colitis in guinea-pigs. J Pharm Pharmacol. 2001;53(12):1711-20. 
[11] Watt J, Marcus SN, Marcus AJ. The comparative prophylactic effects of sulfsalazine, prednisolone, and azathioprine in experimental ulceration. J Pharm Pharmacol. 1980;32(12):873-4.

[12] Kitano A, Matsumoto T, Oshitani N, Nakagawa M, Yasuda K, Watanabe Y, Tomobuchi M, Obayashi M, Tabata A, Fukushima R, Okabe H, Nakamura S, Obata A, Okawa K, Kobayashi K. Distribution and anti-inflammatory effect of mesalazine on carrageenan-induced colitis in the rabbit. Clin Exp Pharmacol Physiol. 1996;23(4):305-9.

[13] Matsumoto T, Kitano A, Oshitani N, Obata A, Hiki M, Hashimura $\mathrm{H}$, Okawa $\mathrm{K}$, Nagura $\mathrm{H}$, Kobayashi $\mathrm{K}$. Immunoglobin-containing cells in the colonic mucosa of rabbits with carrageenan-induced colitis. Dis Colon Rectum. 1986;31(9):723-9.

[14] Jensen BH, Andersen JO, Poulsen SS, Skov Olsen P, Norby Rasmussen S, Hansen SH, Hvidberg EF. The prophylactic effect of 5-aminosalicylic acid and salazosulphapyridine on degraded- carrageenan-induced colitis in guinea pigs. Scand J Gastroenterol. 1984;19(3):299-303.

[15] Utsunomiya I, Nagai S, Ohishi S. Sequential appearance of IL-1 and IL-6 activities in rat carrageenin-induced pleurisy. J Immunol. 1991;147:1803-9.

[16] Talero E, di Paola R, Mazzon E, Esposito E, Motilva V, Cuzzocrea S. Anti-inflammatory effects of adrenomedullin on acute lung injury induced by carrageenan in mice. Mediators Inflamm. 2012;2012:717851.

[17] Borthakur A, Bhattacharyya S, Dudeja PK, Tobacman JK. Carrageenan induces interleukin-8 production through distinct BCL-10 pathway in normal human intestinal epithelial cells. Am J Physiol Gastrointest Liver Physiol. 2007;292(3):G829-38.

[18] Bhattacharyya S, Gill R, Chen M-L, Zhang F, Linhardt RJ, Dudeja PK, Tobacman JK. Toll- like receptor-4 mediates induction of Bcl10-NFkB-IL-8 inflammatory pathway by carrageenan in human intestinal epithelial cells. J Biol Chem. 2008;16(283):10550-8.

[19] Bhattacharyya S, Dudeja PK, Tobacman JK. Carrageenaninduced $\mathrm{NFK}_{\mathrm{B}} \mathrm{B}$ activation depends on distinct pathways mediated by reactive oxygen species and Hsp27 or by Bcl10. Biochim Biophys Acta. 2008;1780(7-8):973-82.

[20] Bhattacharyya S, Borthakur A, Tyagi S, Gill R, Dudeja $\mathrm{PK}$, Tobacman JK. BCL10 is required for NFKB nuclear translocation by both canonical and non-canonical pathways and for NIK phosphorylation. J Biol Chem. 2010;285(1): 522-30.

[21] Baumann BC, Stussi G, Huggel K, Rieben R, Seebach JD. Reactivity of human natural antibodies to endothelial cells from Gal-alpha(1,3)Gal-deficient pigs. Transplantation. 2007;83:193-201.

[22] Galili U. The alpha-gal epitope and the anti-Gal antibody in xenotransplantation and in cancer immunotherapy. Immunol Cell Biol. 2005;83:674-86.

[23] Sandrin MS, Osman N, McKenzie IF. Transgenic approaches for the reduction in expression of GAL- $\alpha(1,3)$ GAL for xenotransplantation. Front Biosci. 1997;2:e1-11.

[24] Tanemura M, Yin D, Chong AS, Galili U. Differential immune responses to alpha-galepitopes on xenografts and allografts: Implications for accommodation in xenotransplantation. Clin Invest. 2000;105:301-10.

[25] Macher BA, Galili U. The Gal-alpha-1,3-Gal-beta-1,4GlcNAc-R (alpha-Gal)epitope: A carbohydrate of unique evolution and clinical relevance. Biochim Biophys Acta. 2008;1780(2):75-88.

[26] West J, Miller KN. California's living marine resources: A status report. Agarophytes and carrageenophytes. California Department of Fish and Game, 2001, pp. 286-287.

[27] "algae." Encyclopedia Britannica. Encyclopedia Britannica Online. Encyclopædia Britannica, 2014. www.britannica.com

[28] Shah ZC, Huffman FG. Current availability and consumption of carrageenan-containing foods. Ecol Food Nutr. 2003; 42:1-15.

[29] Weiner ML. Food additive carrageenan: Part II: A critical review of carrageenan in vivo safety studies. Crit Rev Toxicol. 2014;44(3):244-69.

[30] http://www.consort-statement.org

[31] Walmsley RS, Ayres RC, Pounder RE, Allan RN. A simple clinical colitis activity index. Gut. 1998;43(1):29-32.

[32] www.ClinicalTrials.gov

[33] Jowett SL, Seal CJ, Barton JR, Welfare MR. The short inflammatory bowel disease questionnaire is reliable and responsive to clinically important change in ulcerative colitis. Am J Gastroenterol. 2001;96(10):2921-8.

[34] Seo M, Okada M, Yao T, Okabe N, Maeda K, Oh K. Evaluation of disease activity in patients with moderately active ulcerative colitis: Comparisons between a new activity index and Truelove and Witts' classification. Am J Gastroenterol. 1995;90(10):1759-63.

[35] Truelove SC, Witts LJ. Cortisone in ulcerative colitis. Br Med J. 1955;2(4947):1041-8.

[36] Bhattacharyya S, Pant N, Dudeja PK, Tobacman JK. Development, evaluation, and application of a highly sensitive microtiter plate ELISA for human Bcl10 protein. $\mathbf{J}$ Immunoassay Immunochem. 2007;28(3):173-88.

[37] Li X, LeBlanc J, Elashoff D, McHardy I, Tong M, Roth B, Ippoliti A, Barron G, McGovern D, McDonald K, Newberry R, Graeber T, Horvath S, Goodglick L, Braun J. Microgeographic proteomic networks of the human colonic mucosa and their association with inflammatory bowel disease. Cell Mol Gastroenterol Hepatol. 2016;2(5):567-83.

[38] Sartor RB. Microbial influences in inflammatory bowel disease. Gastroenterology. 2008;134(2):577-94.

[39] Scaldaferri F, Fiocchi C. Inflammatory bowel diseases: Progress and current concepts of etiopathogenesis. J Dig Dis. 2007;8(4):171-8.

[40] Jowett SL, Seal CJ, Pearce MS, Phillips E, Gregory W, Barton $J R$, Welfare MR. Influence of dietary factors on the clinical course of ulcerative colitis: A prospective cohort study. Gut. 2004;53(10):1479-84.

[41] Tragnone A, Valpiani D, Miglio F, Elmi G, Bazzocchi G, Pipitone E, Lanfranchi GA. Dietary habits as risk factors for inflammatory bowel disease. Eur J Gastroenterol Hepatol. 1995;7(1):47-51.

[42] Zvirbliene A, Kiudelis G, Zalinkevicius R, Kupcinskas L. Dietary characteristics of patients with inflammatory bowel disease. Medicina (Kaunas). 2006;42(111):895-9.

[43] Russel MG, Engels LG, Muris JW, Limonard CB, Volovics A, Brummer RJ, Stockbrugger RW. Modern life in the epidemiology of inflammatory bowel disease: A case-control study with special emphasis on nutritional factors. Eur $\mathbf{J}$ Gastroenterol Hepatol. 1998;10(3):243-9.

[44] Sakamoto N, Kono S, Wakai K, Fukuda Y, Satomi M, Shimoyama T, Inaba Y, Miyake Y, Sasaki S, Okamoto 
K, Kobashi G, Washio M, Yokoyama T, Date C, Tanaka $\mathrm{H}$, Epidemiology Group of the Research Committee on Inflammatory Bowel Disease in Japan. Dietary risk factors for inflammatory bowel disease: A multicenter case-control study in Japan. Inflamm Bowel Dis. 2005;11(2):154-63.

[45] Hart AR, Luben R, Olsen A, Tjonneland A, Linseisen J, Nagel G, Berglund G, Lindgren S, Grip O, Key T, Appleby P, Bergmann MM, Boeing H, Hallmans G, Danielsson A, Palmqvist R, Sjodin H, Hagglund G, Overvad K, Palli D, Masala G, Riboli E, Kennedy H, Welch A, Khaw KT, Day N, Bingham S. Diet in the aetiology of ulcerative colitis: A European prospective cohort study. Digestion. 2008;77(1): 57-64.

[46] Reif S, Klein I, Lubin F, Farbstein M, Hallak A, Gilat T. Preillness dietary factors in inflammatory bowel disease. Gut. 1997;40(6):754-60.

[47] Rosman-Urbach M, Niv Y, Birk Y, Morgenstern S, Schwartz B. Relationship between nutritional habits adopted by ulcerative colitis patients relevant to cancer development at clinical remission stages and molecular-genetic parameters. Br J Nutr. 2006;95(1):188-95.

[48] Molodecky NA, Soon IS, Rabi DM, Ghali WA, Ferris M, Chernoff G, Benchimol EI, Panaccione R, Ghosh S, Barkema HW, Kaplan GG. Increasing incidence and prevalence of the inflammatory bowel diseases with time, based on systematic review. Gastroenterology. 2012;142(1):46-54.

[49] World Gastroenterology Organization, WGO Global Guidelines. Inflammatory bowel disease: A global perspective. 2009. www:worldgastroenterology.org

[50] The facts about inflammatory bowel diseases. www.ccfa.org

[51] Ng SC, Bernstein CN, Vatn MH, Lakatos PL, Loftus EV Jr, Tysk C, O'Morain C, Moum B, Colombel JF, Epidemiology and Natural History Task Force of the International Organization of Inflammatory Bowel Disease (IOIBD). Geographical variability and environmental risk factors in inflammatory bowel disease. Gut. 2013;62(4):630-49.

[52] mordorintelligence.com Global carrageenan market - Trends and Forecast. 2014-2019.

[53] Somogyi L, Muller S, Kishi A. Food additives. Specialty Chemicals. SRI consulting, Menlo Park California, 2005.

[54] "Carrageenan." www.cornucopia.org

[55] Kakodkar S, Farooqui AJ, Mikolaitis SL, Mutlu EA. The specific carbohydrate diet for inflammatory bowel disease: A case series. J Acad Nutr Diet. 2015;115:1226-32.

[56] Suskind DL, Wahbeh G, Cohen SA, Damman CJ, Klein J, Braly K, Shaffer M, Lee D. Patients perceive clinical benefit with the specific carbohydrate diet for inflammatory bowel disease. Dig Dis Sci. 2016;61(11):3255-60.

[57] Cohen SA, Gold BD, Oliva S, Lewis J, Stallworth A, Koch B, Eshee L, Mason D. Clinical and mucosal improvement with specific carbohydrate diet in pediatric Crohn disease. J Pediatr Gastroenterol Nutr. 2014;59(4):516-21.

[58] Marlow G, Ellett S, Ferguson IR, Zhu S, Karunasinghe N, Jesuthasan AC, Han DY, Fraser AG, Ferguson LR. Transcriptomics to study the effect of a Mediterranean-inspired diet on inflammation in Crohn's disease patients. Hum Genomics. 2013;7:24.

[59] Panagiotakos D, Kalogeropoulos N, Pitsavos C, Roussinou G, Palliou K, Chrysohoou C, Stefanadis C. Validation of the MedDietScore via the determination of plasma fatty acids. Int J Food Sci Nutr. 2009;60;168-80.

[60] Trichopoulou A, Bamia C, Trichopoulos D. Anatomy of health effects of Mediterranean diet: Greek EPIC prospective cohort study. BMJ. 2009;338:2337. 